\title{
ESTIMATIVAS DE REPETIBILIDADE PARA CARACTERES FORRAGEIROS EM Panicum maximum
}

\author{
Repeatability estimates for forage characters in Panicum maximum \\ Francisco José da Silva Lédo ${ }^{1}$, Antônio Vander Pereira ${ }^{1}$, Fausto de Souza Sobrinho', \\ Alexander Machado Auad ${ }^{1}$, Liana Jank ${ }^{2}$, Jackson Silva e Oliveira ${ }^{3}$
}

\begin{abstract}
RESUMO
Objetiva-se com este trabalho estimar a repetibilidade para caracteres forrageiros de Panicum, e determinar o número de cortes de avaliação necessários para a seleção de genótipos de Panicum, com confiabilidade. Utilizaram-se os dados de um ensaio conduzido no período de 21/11/2002 a 08/04/2005, no Campo Experimental da Embrapa Gado de Leite, localizado em Valença-RJ, onde foram realizados 15 cortes de avaliação. No ensaio, foram avaliados 23 genótipos de Panicum maximum, em parcelas experimentais, dispostas no delineamento de blocos casualizados, com três repetições. Foram estimados os coeficientes de repetibilidade para as características produção de matéria verde de forragem (PMV); produção de matéria seca de forragem (PMS) e de folhas (PMSF); porcentagem de folhas na PMS (\%FOL) e altura da planta (AP), utilizando os métodos da análise de variância, componentes principais e análise estrutural. Para todas as características avaliadas os efeitos de genótipos, cortes e interação genótipos x cortes foram significativos $(\mathrm{P}<0,01)$. Quando se considerou o coeficiente de determinação de $85 \%$, o número de avaliações (cortes) necessários para a determinação do valor real dos genótipos foram de 10, 9, 7, 11 e 3, respectivamente, para PMV, PMS, PMSF, \%FOL e AP. O método dos componentes principais e o da análiseestrutural (baseado na matriz de correlações) foram concordantes para todas as características avaliadas. A realização de 10 cortes de avaliação permite discriminar o valor real dos genótipos de Panicum, com confiabilidade superior a $85 \%$, para a maioria das características avaliadas.
\end{abstract}

Termos para indexação: Forrageira, gramínea, componentes principais, análise estrutural, coeficiente de repetibilidade.

\section{ABSTRACT}

The objective of this work was to estimate the repeatability for forage characters of Panicum and to determinate the necessary number of evaluation cuts to select Panicum genotypes with confidence. Data of a trial with 15 cuts, carried out between 21/11/2002 and 08/04/2005 in the experimental station of Embrapa Gado de Leite located in Valença, RJ, Brazil, were used. In this study. 23 genotypes of "Panicum maximum" were evaluated, in a complete randomized block, with three replications. The coefficient of repeatability for fresh forage production (PMV), total plant dry matter production (PMS) and leaves dry matter production (PMSF) were recorded along with leaves percentage in PMS (\%FOL) and plant hight (AP), using the variance analysis, main components and structural analysis methods. For all evaluated parameters the effects of genotype, cut and genotype $\mathrm{x}$ cut interaction were significant $(\mathrm{P}<0.01)$. When considering the determination coefficient as $85 \%$, the required number of measures (cuts) to determine the real value of genotypes were 10, 9, 7, 11 and 3, for PMV, PMS, PMSF, \%FOL and AP, respectively. Main components and structural analysis methods (based on the correlation matrix) did agree for all features evaluated. The utilization of data from 10 cuts allows to discriminate, with confidence higher than $85 \%$, the real value for most of the evaluated characters of Panicum genotypes.

Index terms: Pasture, grass, main components, structural analysis, repeatability coefficient.

(Recebido em 3 de outubro de 2006 e aprovado em 15 de fevereiro de 2007)

\section{INTRODUÇÃO}

A intensificação dos sistemas de produção de leite exige a utilização de forrageiras que combinem elevada capacidade de produção com alta qualidade (PEREIRA et al., 2001). Forrageiras do gênero Panicum constituem boa opção para animais de maior potencial genético, já que, em geral, apresentam maior valor nutritivo quando comparadas com as braquiárias. Também na produção de leite, e não só no Brasil, o Panicum maximum tem se revelado importante, seja para pastejo direto ou para silagem e feno (ESPERANCE \& DIAZ, 1985; HACKER \& JANK, 1998).

No programa de melhoramento genético de Panicum, coordenado pela Embrapa Gado de Corte, inicialmente os genótipos mais promissores são avaliados por meio de ensaios em rede, onde são submetidos a cortes

\footnotetext{
Engenheiros Agrônomos, Doutores - Embrapa Gado de Leite - Rua Eugênio do Nascimento, 610, Dom Bosco - $36038-330$ - Juiz de Fora, MG ledo@cnpgl.embrapa.br; avanderp@cnpgl.embrapa.br; fausto@cnpgl.embrapa.br; amauad@cnpgl.embrapa.br

²Engenheira Agrônoma, Phd-Embrapa Gado de Corte-Br 262, Km 04, Zona Rural - Cx. P. 154-79002-970 - Campo Grande, MS - liana@cnpgc.embrapa.br 3Engenheiro Agrônomo, Phd - Embrapa Gado de Leite - Rua Eugênio do Nascimento, 610, Dom Bosco - $36038-330$ - Juiz de Fora, MG jackoliv@cnpgl.embrapa.br
} 
sucessivos de avaliação. Apenas os melhores genótipos selecionados nessa etapa, serão, posteriormente, avaliados sob condição de pastejo. Nessa situação, ou mesmo em outros ensaios de avaliação de cultivares de Panicum sob condição de corte, é necessário que o número de avaliações (cortes) sejam suficientes para permitir a seleção, com confiabilidade, dos melhores genótipos. Segundo SouzaSobrinho et al. (2004), essa também é uma dificuldade encontrada no melhoramento de forrageiras, já que é necessário realizar um número mínimo de avaliações para se detectar diferenças entre genótipos.

A repetibilidade expressa a proporção da variância total, que é explicada pelas variações proporcionadas pelos genótipos e pelas alterações pemanentes atribuídas ao ambiente comum (CRUZ \& REGAZZI, 1997), e através do seu estudo é possível estimar o número de medições necessárias para predizer o valor real de um genótipo. Existem diferentes métodos que permitem estimar os coeficientes de repetibilidade, como o da análise de variância, componentes principais e análise estrutural (ABEYWARDENA, 1972; CRUZ \& REGAZZI, 1997; MANSOUR et al., 1981), que vêm sendo utilizados em estudos de repetibilidade, em diferente culturas, dentre elas o capim-elefante (SHIMOYA et al., 2002), alfafa (SOUZA-SOBRINHO et al., 2004) e a cana-de açúcar (FERREIRA et al., 2005; SANTOS et al., 2004).

Objetivou-se, neste trabalho estimar a repetibilidade para caracteres forrageiros de Panicum, e determinar o número de avaliações necessários para a seleção de genótipos de Panicum, com confiabilidade.

\section{MATERIAL E MÉTODOS}

O ensaio foi conduzido no Campo Experimental Santa Mônica da Embrapa Gado de Leite, localizado em Valença-RJ. Foram avaliados 23 genótipos de Panicum maximum, sendo 14 acessos do Banco de Germoplasma, 4 híbridos intra-específicos e cinco cultivares comerciais (Tanzânia-1, Mombaça, Massai, Milênio e Aruana). Os acessos e híbridos foram selecionados pelo programa de melhoramento genético de Panicum da Embrapa Gado de Corte.

As parcelas experimentais foram dispostas no delineamento de blocos casualizados, com três repetições. Cada parcela foi constituída de seis linhas de $4 \mathrm{~m}$, com espaçamento entre linhas de $0,5 \mathrm{~m}$. Todas as parcelas foram distanciadas uma das outras em $2 \mathrm{~m}$. Como após a implantação do ensaio, constatou-se que muitas parcelas apresentavam falhas, foi realizado um corte em 21/01/2003, onde foram corrigidas as falhas nas fileiras (replantio) utilizando mudas retiradas das bordaduras da parcela. O corte de uniformização foi realizado em 09/04/2003, em seguida realizaram-se quinze cortes de avaliação (11/06/03, 30/09/03, 05/11/03, 16/12/03, 29/01/04, 04/03/04, 07/04/04, 13/ 05/04, 11/08/04, 20/10/04, 23/11/04, 20/12/2004, 02/02/2005, 02/03/2005 e 08/04/2005), procurando manter intervalos entre cortes de cinco semanas na época chuvosa. $\mathrm{Na}$ época seca foram realizados apenas dois cortes.

As características avaliadas foram: produção de matéria verde de forragem (PMV); produção de matéria seca de forragem (PMS) e de folhas (PMSF); porcentagem de folhas na PMS (\%FOL) e altura da planta (AP). A PMSF e \%FOL não foram avaliadas no primeiro e segundo cortes de avaliação; nesse caso, o estudo de repetibilidade considerou 15 avaliações (cortes) para as características PMV, PMS e AP, e 13 avaliações para PMSF e \%FOL.

Antes do estudo de repetibilidade, foi realizada análise de variância para todas as características considerando o modelo de parcela subdividida no tempo, em que os genótipos eram as parcelas, e os cortes de avaliação as subparcelas (RAMALHO et al., 2000). Apenas nas características em que foram constatadas diferenças significativas entre os genótipos $(\mathrm{P}<0,05)$, foi realizado o estudo de repetibilidade. $\mathrm{Na}$ estimativa do coeficiente de repetibilidade pelo método da análise de variância (Anova), utilizou-se a metodologia descrita por Cruz \& Regazzi (1997), considerando o modelo $\mathrm{Y}_{\mathrm{ij}}=\mathrm{m}+\mathrm{G}_{\mathrm{i}}+\mathrm{C}_{\mathrm{j}}+\mathrm{E}_{\mathrm{ij}}$, em que $\mathrm{Y}_{\mathrm{ij}}$ : média da i-ésimo genótipo no j-ésimo corte; $\mathrm{m}$ : média geral; $\mathrm{G}_{\mathrm{i}}$ : efeito aleatório do i-ésimo genótipo sob influência do ambiente permanente (corte); $C_{j}$ : efeito fixo do ambiente temporário no j-ésimo corte; $\mathrm{E}_{\mathrm{ij}}$ : erro experimental estabelecido pelos efeitos temporários no jésimo corte aleatório que envolve outras causas de variação, não incluídas no modelo. Também foram estimados os coeficientes de repetibilidade pelos métodos dos componentes principais obtidos da matriz de variâncias e covariâncias (CP-var) e da matriz de correlação (CP-cor); e pela análise estrutural, com base no autovalor teórico da matriz de correlações (AE-cor), conforme metodologia descrita em Cruz \& Regazzi (1997), onde na obtenção da matriz pelos métodos $\mathrm{CP}$-var e $\mathrm{CP}$-cor, foram consideradas as medidas realizadas no tempo, como diferentes variáveis. Foram estimados também, o número mínimo de medições necessárias para predizer o valor real dos genótipos, com base em diferentes coeficientes de determinação $\left(R^{2}\right)$ préestabelecidos (80, 85, 90, 95 e 99\%), calculado segundo Cruz \& Regazzi (1997), por meio da expressão

$$
\eta_{0}=\frac{R^{2}(1-r)}{\left(1-R^{2}\right) r}
$$


em que $\eta_{0}$ : número mínimo de medições para predição do valor real; : coeficiente de determinação; e : coeficiente de repetibilidade.

\section{RESULTADOS E DISCUSSÃO}

Para todas as características avaliadas os efeitos de genótipos, cortes e interação genótipos x cortes foram significativos $(\mathrm{P}<0,01)$, indicando que os genótipos de Panicum apresentaram diferenças entre si em todas as características avaliadas, e de que o comportamento relativo deles não foi o mesmo em todos os cortes, ou seja, o genótipo de melhor desempenho, num dado corte, pode não ter apresentado esse comportamento em um outro. Esses resultados reforçam a importância do estudo de repetibilidade, para se determinar o número mínimo de cortes necessários para a predição do valor real dos genótipos de Panicum.

As magnitudes das estimativas dos coeficientes de repetibilidade variaram entre as características avaliadas (Tabela 1). Esse resultado era esperado, já que o fenótipo apresentado por um caráter resulta da ação do genótipo, sob influências do meio, podendo em alguns casos, ser regulado por conjuntos gênicos diferentes (CRUZ \& REGAZZI, 1997). Com exceção da altura da planta (AP), todas as demais características apresentaram estimativas de coeficientes de repetibilidade variando de 0,167 a 0,542 , indicando que não houve regularidade na repetição do desempenho dos genótipos de uma avaliação para outra, o que é corroborado pela interação genótipo $\mathrm{x}$ corte significativo. Entretanto, como se tratam de características quantitativas, e de uma espécie perene, a magnitude destas estimativas podem ser consideradas razoáveis, uma vez que a predição do valor real, que é expressa pelo coeficiente de determinação, ficaram acima de 72,32\%.

Para as características: produção de matéria seca de forragem de folhas (PMSF) e altura da planta (AP), os quatro métodos utilizados nas estimativas dos coeficientes de repetibilidade foram concordantes entre si, e apresentaram valores médios de 0,478 e 0,663, respectivamente para PMSF e AP. Em trabalhos realizados com cana-de-açúcar (FERREIRA et al., 2005), capimelefante (SHIMOYA et al., 2002) e alfafa (SOUZASOBRINHO et al., 2004) também verificaram-se que os métodos da análise de variância, componentes principais e análise estrutural, proporcionaram estimativas bem semelhantes. Já para a produção de matéria verde de forragem (PMV), produção de matéria seca de forragem (PMS) e porcentagem de folhas na PMS (\%FOL) o método da Anova apresentou estimativas de 0,253; 0,231 e 0,167; respectivamente para PMV, PMS e \%FOL, consideradas discrepantes das obtidas pelos métodos $\mathrm{CP}$-cov, $\mathrm{CP}$-cor e $\mathrm{AE}$-cor, que tiveram valores médios de 0,371; 0,384 e 0,357, respectivamente para PMV, PMS e \%FOL. Assim, apenas o método dos componentes principais e o da análise estrutural foram concordantes, para todas as características avaliadas.

As estimativas dos coeficientes de repetibilidade, obtidas para PMS e AP, foram próximas dos resultados verificados no trabalho de Carvalho Filho et al. (2004), realizado também com Panicum (média para os quatro

Tabela 1 - Estimativas dos coeficientes de repetibilidade e respectivos coeficientes de determinação (entre parêntesis) da produção de matéria verde de forragem (PMV); produção de matéria seca de forragem (PMS) e de folhas (PMSF); porcentagem de folhas na PMS (\%FOL) e altura da planta (AP), obtidas por diferentes metodologias de estimação.

\begin{tabular}{lccccc}
\hline & \multicolumn{5}{c}{ Características } \\
\cline { 2 - 6 } Metodologia de estimação & PMV & PMS & PMSF & \%FOL & AP \\
\hline $\begin{array}{l}\text { Análise de variância } \\
\text { (Anova) }\end{array}$ & 0,253 & 0,231 & 0,412 & 0,167 & 0,603 \\
$\begin{array}{l}\text { Componentes principais - variâncias } \\
\text { e covariâncias (CP-cov) }\end{array}$ & $(83,58)$ & $(81,83)$ & $(90,09)$ & $(72,32)$ & $(95,80)$ \\
& $(88,49)$ & $(91,38)$ & $(93,90)$ & $(88,39)$ & $(97,80)$ \\
$\begin{array}{l}\text { Componentes principais - } \\
\text { correlação (CP-cor) }\end{array}$ & 0,401 & 0,386 & 0,491 & 0,392 & 0,659 \\
$\begin{array}{l}\text { Análise estrutural - } \\
\text { correlação (AE-cor) }\end{array}$ & $(90,93)$ & $(90,41)$ & $(92,61)$ & $(89,35)$ & $(96,66)$ \\
& 0,372 & 0,353 & 0,468 & 0,309 & 0,642 \\
& $(89,87)$ & $(89,10)$ & $(91,94)$ & $(85,34)$ & $(96,42)$ \\
\hline
\end{tabular}


métodos de 0,313 e 0,683, respectivamente para PMS e AP), entretanto, para a PMSF as estimativas obtidas foram inferiores (média para os quatro métodos de 0,633). Carvalho Filho et al. (2004) utilizaram dados de um ensaio que possuia apenas 5 genótipos de Panicum e 8 cortes de avaliação, o que é considerado baixo, além de utilizar dados em que o efeito de genótipo foi não significativo $(\mathrm{P}>0,05)$, o que pode explicar as diferenças verificadas nas estimativas dos coeficientes de repetibilidade para a PMSF.

Para a PMSF, os coeficientes de determinação associados às estimativas dos coeficientes de repetibilidade obtidos pelas quatro metodologias de estimação, variaram de 90,09 a 93,90\% indicando que as 13 avaliações (cortes) realizadas permitiram predizer o valor real dos genótipos, com determinação acima de $90 \%$, enquanto que para a \%FOL, esses valores variaram de
72,32 a 89,35\%. Já para PMV, PMS e AP, onde foram realizadas 15 avaliações, e os coeficientes de determinação obtidos pelos métodos Anova, CP-cov, CP-cor e AE-cor variaram de 83,58 a 90,93\% para PMV; 81,83 a 91,38\% para PMS; e 95,80 a $97,80 \%$ para AP.

O número de medições necessárias para se atingir coeficientes de determinação $\left(R^{2}\right)$ de 80 a 99, considerando quatro metodologias de estimação, estão apresentados na Tabela 2. A realização de 10 e 5 avaliações (média do número de medições obtidas pelas quatro metodologias), respectivamente para PMSF e AP, permitiu discriminar os genótipos com $90 \%$ de certeza de estar avaliando o valor real de cada um deles, enquanto que 7 e 3 avaliações, respectivamente para PMSF e AP, permite confiabilidade de $85 \%$. Considerando apenas a média do número de medições obtidos pelos métodos $\mathrm{CP}$-cov, $\mathrm{CP}$-cor e $\mathrm{AE}$-cor,

Tabela 2 - Números de medições (cortes) necessárias para diferentes coeficientes de determinação $\left(\mathrm{R}^{2}\right)$, para as características: produção de matéria verde de forragem (PMV); produção de matéria seca de forragem (PMS) e de folhas (PMSF); porcentagem de folhas na PMS (\%FOL) e altura da planta (AP), obtidas por diferentes metodologias de estimação.

\begin{tabular}{|c|c|c|c|c|c|c|}
\hline \multirow{2}{*}{ Metodologia de estimação } & \multirow{2}{*}{$R^{2}(\%)$} & \multicolumn{5}{|c|}{ Características } \\
\hline & & PMV & PMS & PMSF & $\% \mathrm{FOL}$ & $\mathrm{AP}$ \\
\hline \multirow{5}{*}{$\begin{array}{l}\text { Análise de variância } \\
\text { (Anova) }\end{array}$} & 80 & 12 & 13 & 6 & 20 & 3 \\
\hline & 85 & 17 & 19 & 8 & 28 & 4 \\
\hline & 90 & 27 & 30 & 13 & 45 & 6 \\
\hline & 95 & 56 & 63 & 27 & 95 & 12 \\
\hline & 99 & 292 & 330 & 142 & 493 & 65 \\
\hline \multirow{5}{*}{$\begin{array}{l}\text { Componentes principais - } \\
\text { variâncias e covariâncias } \\
\text { (CP-cov) }\end{array}$} & 80 & 8 & 6 & 3 & 7 & 1 \\
\hline & 85 & 11 & 8 & 5 & 10 & 2 \\
\hline & 90 & 18 & 13 & 8 & 15 & 3 \\
\hline & 95 & 37 & 27 & 16 & 32 & 6 \\
\hline & 99 & 193 & 140 & 84 & 169 & 33 \\
\hline \multirow{5}{*}{$\begin{array}{l}\text { Componentes principais - } \\
\text { correlação (CP-cor) }\end{array}$} & 80 & 6 & 6 & 4 & 6 & 2 \\
\hline & 85 & 8 & 9 & 6 & 9 & 3 \\
\hline & 90 & 13 & 14 & 9 & 14 & 5 \\
\hline & 95 & 28 & 30 & 20 & 29 & 10 \\
\hline & 99 & 148 & 158 & 103 & 153 & 51 \\
\hline \multirow{5}{*}{$\begin{array}{l}\text { Análise estrutural - } \\
\text { correlação (AE-cor) }\end{array}$} & 80 & 7 & 7 & 5 & 9 & 2 \\
\hline & 85 & 10 & 10 & 6 & 13 & 3 \\
\hline & 90 & 15 & 17 & 10 & 20 & 5 \\
\hline & 95 & 32 & 35 & 22 & 42 & 11 \\
\hline & 99 & 167 & 182 & 113 & 221 & 55 \\
\hline
\end{tabular}


a realização de 16,15 e 17 avaliações, respectivamente para PMV, PMS e \%FOL, permite a seleção com confiabilidade de $90 \%$. Entretanto, para atingir um coeficiente de determinação de $85 \%$, o número de cortes necessários foi de 10, 9 e 11, respectivamente para PMV, PMS e \%FOL.

Para as características ligadas à produção de forragem (PMV, PMS, PMSF e \%FOL), a necessidade de se ter um elevado número de cortes, que permita a seleção com confiabilidade dos melhores genótipos, já era esperada, uma vez que se trata de uma espécie perene, que apresenta grande diversidade genética (PEREIRA et al., 2001), e cuja produtividade de forragem é muito influenciada pelas variações climáticas que ocorrem ao longo do ano, principalmente temperatura e precipitação pluviométrica (HEINEMANN et al., 2005).

Com base nas estimativas de repetibilidade, e considerando uma confiabilidade de $85 \%$, recomenda-se que, em ensaios de avaliação e seleção de genótipos de Panicum, sob condição de corte, sejam realizados pelo menos 10 cortes de avaliação, para que se tenha confirmado o potencial forrageiro do genótipo selecionado. Entretanto, deve-se ressaltar que, dependendo das condições edafoclimáticas, do conjunto de genótipos avaliados, e do objetivo do trabalho, o número de avaliações necessárias para realizar a seleção, com confiabilidade, pode ser superior a 10 cortes.

\section{CONCLUSÕES}

A realização de 10 cortes de avaliação permite seleção adequada de genótipos de Panicum maximum, com confiabilidade superior a $85 \%$, para a maioria das características avaliadas.

O método dos componentes principais (obtidos da matriz de variâncias e covariâncias, e da matriz de correlação) e o método da análise estrutural, com base no autovalor teórico da matriz de correlações, foram concordantes para todas as características avaliadas.

\section{REFERÊNCIAS BIBLIOGRÁFICAS}

ABEYWARDENA, V. An application of principal component analysis in genetics. Journal of Genetics, Bangalore, v. 16, p. 27-51, 1972.

CARVALHO FILHO, A.; CASTILHOS, Z. M. S.; STORCK, L. Análise de repetibilidade de caracteres forrageiros de genótipos de Panicum maximum, avaliados com e sem restrição solar. Ciência Rural, Santa Maria, v. 34, n. 3, p. 723-729, maio/jun. 2004.

CRUZ, C. D.; REGAZZI, A. J. Modelos biométricos aplicados ao melhoramento genético. 2. ed. Viçosa: UFV, 1997. $390 \mathrm{p}$.
ESPERANCE, M.; DIAZ, D. Valor nutritivo y producción de leche en los ensilajes sin miel de guinea likoni, pasto estrella y king grass. Pastos y Forages, Matanzas, v. 8, p. 297-305, 1985.

FERREIRA, A.; BARBOSA, M. H. P.; CRUZ, C. D.; HOFFMANN, H. P.; VIEIRA, M. A. S.; BASSINELLO, A. I.; SILVA, M. F. Repetibilidade e número de colheitas para seleção de clones de cana-de-açúcar. Pesquisa Agropecuária Brasileira, Brasília, v. 40, n. 8, p. 761-767, ago. 2005.

HACKER, J. B.; JANK, L. Breeding tropical and subtropical. In: CHERNEY, J. H.; CHERNEY, D. J. R. (Eds.). Grass for dairy cattle. Cambridge: CABI, 1998. p. 49-72.

HEINEMANN, A. B.; FONTES, A. J.; PACIULLO, D. S. C.; ROSA, B.; MACEDO, R.; MOREIRA, P.; AROEIRA, L. J. M. Potencial produtivo e composição bromatológica de seis gramíneas forrageiras tropicais, sob duas doses de nitrogênio e potássio. Pasturas Tropicales, Cali, v. 27, n. 1, 2005.

PEREIRA, A. V.; VALLE, C. B.; FERREIRA, R. de P.; MILES, J. W. Melhoramento de forrageiras tropicais. In: NASS, L. L.; VALOIS, A. C. C.; MELO, I. S.; VALADARES-INGRES, M. C. (Eds.). Recursos genéticos e melhoramento de plantas. Rondonópolis: Fundação Mato Grosso, 2001. p. 549-602.

MANSOUR, H.; NORDHEIM, E. V.; RULEDGE, J. J. Estimators of repeatability. Theoretical and Applied Genetics, Heidelberg, v. 60, p. 151-156, 1981.

RAMALHO, M. A. P.; FERREIRA, D. F.; OLIVEIRA, A. C. Experimentação em genética e melhoramento de plantas. Lavras: UFLA, 2000. 326 p.

SANTOS, M. S. M.; MADALENA, J. A.; SOARES, L.; FERREIRA, P. V.; BARBOSA, G. V. S. Repetibilidade de características agroindustriais em cana-de-açúcar. Pesquisa Agropecuária Brasileira, Brasília, v. 39, n. 4, p. 301-306, abr. 2004.

SHIMOYA, A.; PEREIRA, A. V.; FERREIRA, R. de P.; CRUZ, C. D.; CARNEIRO, P. C. S. Repetibilidade de características forrageiras do capim-elefante. Scientia Agrícola, Piracicaba, v. 59, n. 2, p. 227-234, abr./jun. 2002.

SOUZA-SOBRINHO, F.; LEDO, F. J. S.; PEREIRA, A. V.; BOTREL, M. A.; EVANGELISTA, A. R.; VIANA, M. C. M. Estimativas de repetibilidade para produção de matéria seca em alfafa. Ciência Rural, Santa Maria, v. 34, n. 2, p. 531537, mar./abr. 2004. 